\title{
Predictive value of overt and non-overt volume overload in patients with high- or low-gradient aortic stenosis undergoing transcatheter aortic valve implantation
}

\author{
Ulrich Fischer-Rasokat ${ }^{1}$, Matthias Renker ${ }^{1,2,3}$, Christoph Liebetrau ${ }^{1,3,4}$, Maren Weferling ${ }^{1,3}$, \\ Andreas Rieth ${ }^{1,3}$, Andreas Rolf ${ }^{1,3,5}$, Yeong-Hoon Choi ${ }^{2}$, Christian W. Hamm ${ }^{1,3,5}$, Won-Keun Kim ${ }^{1,2,3,5}$ \\ ${ }^{1}$ Department of Cardiology, Kerckhoff Heart Center, Bad Nauheim, Germany; ${ }^{2}$ Cardiac Surgery, Kerckhoff Heart Center, Bad Nauheim, Germany; \\ ${ }^{3}$ German Centre for Cardiovascular Research (DZHK), Partner Site RheinMain, Bad Nauheim, Germany; ${ }^{4}$ Cardioangiological Center Bethanien \\ (CCB), Frankfurt, Germany; ${ }^{5}$ Medical Clinic I (Cardiology and Angiology), University Hospital of Giessen, Giessen, Germany \\ Contributions: (I) Conception and design: U Fischer-Rasokat, C Hamm, WK Kim; (II) Administrative support: A Rieth, A Rolf, YH Choi, C Hamm; \\ (III) Provision of study materials or patients: U Fischer-Rasokat, M Renker, C Liebetrau, M Weferling, A Rieth, WK Kim, YH Choi; (IV) Collection \\ and assembly of data: M Renker, C Liebetrau, WK Kim; (V) Data analysis and interpretation: All authors; (VI) Manuscript writing: All authors; (VII) \\ Final approval of manuscript: All authors. \\ Correspondence to: Ulrich Fischer-Rasokat. Kerckhoff Heart Center, Benekestr. 2-8, 60231 Bad Nauheim, Germany. \\ Email: u.fischer-rasokat@kerckhoff-klinik.de.
}

Background: The plasma volume status (PVS) is considered a marker of non-overt cardiac congestion and is of prognostic value. Patients with low-flow, low-gradient (LFLG) aortic stenosis (AS) suffer from impaired left ventricular function and show signs of heart failure (HF). We hypothesized that PVS might predict postinterventional rehospitalization and cardiovascular mortality in high-risk patients undergoing transcatheter aortic valve implantation (TAVI).

Methods: In this retrospective, observational analysis, PVS before transfemoral TAVI was calculated by a formula taking into account hematocrit and weight. The predictive performance of PVS was compared with that of prior cardiac decompensation (PCD).

Results: In the entire cohort of $\mathrm{n}=2,458$ patients, PVS >-4\% (high plasma volume) identified patients $(n=1,013)$ with a higher post-interventional 1 -year mortality rate than patients $(n=1,445)$ with a PVS $\leq-4 \%$ (low plasma volume). However, PVS lost prognostic independence when adjusted for anemia, whereas PCD did not. A high PVS and PCD were not correlated, and both parameters similarly revealed a low sensitivity and specificity but a high negative predictive value (NPV) for future HF events. PVS was not different between control patients $(\mathrm{n}=1,512)$ and those with intermediate (paradoxical LFLG-AS, $\mathrm{n}=327$ ) or high risk scores (LFLG-AS, $n=239$ ). The accuracy of high PVS in predicting adverse events in these subpopulations was the same as in the study population overall. Kaplan-Maier analyses demonstrated similar prognostic impacts for PVS and PCD.

Conclusions: PVS and PCD represent two independent parameters of volume overload with unfavorable prognostic significance. Pre-interventional PVS does not appear to be suitable for predicting clinical outcomes in high-risk patients undergoing TAVI.

Keywords: Transcatheter aortic valve implantation (TAVI); plasma volume status (PVS); heart failure (HF); lowflow low-gradient aortic stenosis (LFLG-AS)

Submitted May 03, 2021. Accepted for publication Jun 08, 2021.

doi: $10.21037 / \mathrm{cdt}-21-286$

View this article at: https://dx.doi.org/10.21037/cdt-21-286 


\section{Introduction}

Worsening of heart failure (HF) is the most common reason for readmission after transcatheter aortic valve implantation (TAVI), and rates of re-hospitalization for $\mathrm{HF}$ range from $16 \%$ at 30 days (1) to $24 \%$ at one year (2) post-TAVI, reducing the positive overall costbenefit relationship of TAVI procedures in certain patient population (3). Moreover, patients with severe aortic stenosis (AS) and concomitant systolic HF continue to have severely limited outcomes after TAVI (4), indicating that persisting left ventricular impairment drives prognosis even after correction of afterload. Worsening of HF marks a crossroads in the course of HF. Once pre-existing HF manifests with new or worsening symptoms and signs, the subsequent risk of death skyrockets and remains elevated by up to 3.5 folds, even more than 18 months thereafter (5). Volume overload in HF patients does not necessarily manifest as overt hemodynamic overload (peripheral or pulmonary edema) but may also occur without clinical signs as covert congestion in apparently euvolemic patients. A marker of non-overt volume overload is the plasma volume status (PVS), which reflects the patient's actual deviation from the ideal plasma volume. This parameter can easily be calculated based on weight and hematocrit (6). A high PVS (indicating fluid overload) can precede overt clinical congestion and has emerged as an independent predictor of mortality in chronic HF patients (6). PVS predicted higher rates of postoperative complications and mortality in patients undergoing coronary bypass surgery (7) and was associated with early adverse events post-TAVI (8-10). Patients with low-flow, low-gradient AS and reduced (LFLG-AS) or preserved (paradoxical LFLG-AS) ejection fraction $(\mathrm{EF})$ present with an impaired left ventricular function that is reflected by low stroke volumes and are characterized by a high cardiovascular risk and a high burden of manifest cardiovascular disease (11). Currently it is not completely understood why outcomes are markedly worse in patients with LFLG-AS compared with "classical" high-gradient AS patients, even when baseline parameters of the two groups are matched (11). We speculated that these patients might have subclinical cardiac congestion, given that they per se could be diagnosed to have concomitant $\mathrm{HF}$, and that PVS might be a useful tool to identify those patients at risk of post-procedural cardiac decompensation. Therefore, we assessed PVS in a population of patients undergoing TAVI and in a subset with LFLG-AS and evaluated the performance of PVS in predicting cardiovascular events in these patient cohorts compared with the predictive value of prior cardiac decompensation (PCD).

We present the following article in accordance with the STROBE reporting checklist (available at https://dx.doi. org/10.21037/cdt-21-286).

\section{Methods}

Patients who underwent TAVI for symptomatic, severe AS (aortic valve area index $<0.6 \mathrm{~cm}^{2} / \mathrm{m}^{2}$ ) at a single, highvolume center between January 2011 and May 2020 were included consecutively in this retrospective, observational registry. Only patients with transfemoral access routes were included. All patients were discussed in an interdisciplinary heart team, and decisions were made in adherence to existing guidelines. This included the selection of the type and size of the implanted prostheses. All procedures were performed in a hybrid operation room or in a dedicated catheterization laboratory. General anesthesia with guidance by transesophageal echocardiography was the standard until mid-2014; thereafter, procedures were performed under conscious sedation using transthoracic echocardiography for peri-procedural evaluation of paravalvular regurgitation. Valve deployment was performed with or without rapid pacing according to the type of prosthesis and the operators' decision. Procedural success was evaluated as defined by Valvular Academic Research Consortium-2 (VARC-2) (12). The study was conducted in accordance with the Declaration of Helsinki (as revised in 2013). The study was approved by institutional ethics committee of the University of Giessen (No. AZ 01/21), and individual consent for this retrospective analysis was waived.

The flowchart for the creation of the study populations is depicted in Figure 1: (I) an overall study population was built for the exploratory analysis of high vs. low PVS; (II) patients were alternatively classified into high-gradient patients [HG-AS/controls, mean pressure gradient (MPG) $\geq 40 \mathrm{mmHg}$ ] and into patients with LFLG-AS [MPG $<40 \mathrm{mmHg}$, stroke volume index (SVI) $\leq 35 \mathrm{~mL} / \mathrm{m}^{2}$, EF $<50 \%$ ] or pLFLG-AS (same as LFLG-AS but EF $\geq 50 \%$ ) as described before (11). PCD was defined as an event of hospitalization due to fluid overload and accompanying dyspnea before admission for TAVI as documented in the medical history of the patient or reported by the patient at the time of admission. The primary endpoint was death from any cause within one year post-intervention. Patients with follow-up time longer than one year were censored as 


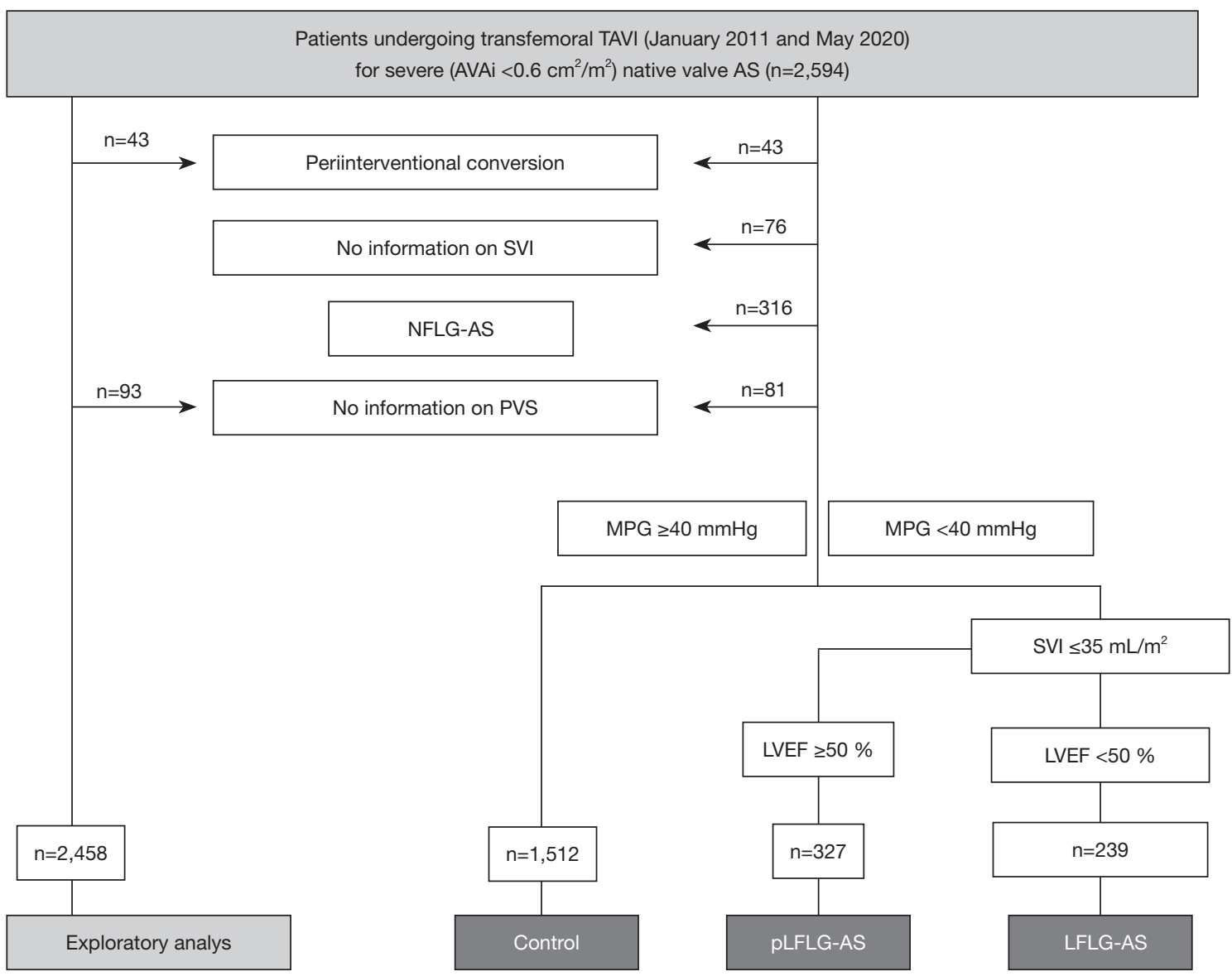

Figure 1 Flow chart illustrating the creation of the study populations. TAVI, transcatheter aortic valve implantation; AVAi, aortic valve area index; AS, aortic stenosis; SVI, stroke volume index; NFLG-AS, normal-flow low-gradient aortic stenosis; PVS, plasma volume status; MPG, mean pressure gradient; LVEF, left ventricular ejection fraction; pLFLG-AS, paradoxical LFLG-AS; LFLG-AS, low-flow lowgradient AS.

alive after 365 days. Patients with loss to follow-up were also censored as alive at the day of last follow-up. Cardiovascular death that occurred post-TAVI, within the 30-day or 1-year follow-up, was defined according to the Valve Academic Research Consortium-2 consensus document (12). Cardiac decompensation post-TAVI was defined according to the definition of PCD.

Actual plasma volume (aPV) was calculated according to an equation that had been validated against measured plasma volumes by the gold-standard radiolabeled albumin assay as (6): $\mathrm{aPV}=(1$ hematocrit $) \times\{\mathrm{a}+[\mathrm{b} \times$ weight $(\mathrm{kg})]\}$

where hematocrit is a fraction, and $a=1,530$ in males and 864 in females, and $b=41$ in males and 47.9 in females.

Ideal plasma volume ( $\mathrm{PPV}$ ) was calculated as described (13): ideal PV $(\mathrm{iPV})=\mathrm{c} \times$ weight $(\mathrm{kg})$ where $\mathrm{c}=39$ in males and 40 in females.

The PVS reflects the divergence of the aPV from iPV in percent (6) (with higher values indicating volume overload):

$\mathrm{PVS}=[($ actual PV - ideal PV $) /$ ideal PV $] \times 100 \%$

In the Val-HeFT cohort consisting of 5,000 patients with chronic HF, PVS >-4\% was demonstrated to be associated with increased mortality, and the independent prognostic impact was confirmed in an outpatient population of patients with chronic HF (6). Therefore, patients in our study were further dichotomized into those with PVS $\leq$ or $>-4 \%$.

Based on a very recent analysis of data obtained from the Treatment of Preserved Cardiac Function Heart Failure with an Aldosterone Antagonist (TOPCAT) trial (14), which suggested that an alternatively estimated PV published 
by Duarte et al. (15) might be of better prognostic utility than the aforementioned formula to predict cardiovascular adverse events in patients with $\mathrm{HF}$ and preserved EF, we additionally calculated the $\mathrm{PV}_{\text {Duarte }}$ as:

$$
\mathrm{PV}_{\text {Duarte }}(\mathrm{mL} / \mathrm{g})=100 \times(1-\text { hematocrit }) / \text { hemoglobin }(\mathrm{g} / \mathrm{dL})
$$

\section{Statistical analysis}

Continuous data are reported as median and interquartile range (IQR). Continuous values were compared by the Mann-Whitney Kruskal-Wallis test and categorical variables by the $\chi^{2}$ test. The test statistics calculated in the analysis of the predictive value of PVS >-4\% included sensitivity $(\mathrm{Sn})$, specificity (Sp), and positive (PPV) and negative predictive values (NPV). Pearson's $\varphi$ coefficient was used to analyze the correlation between PVS $\leq$ or $>-4 \%$ and the prevalence of PCD. To analyze the prediction of a high PVS, all baseline parameters (no procedural parameters) from Table 1 with significant $(\mathrm{P}<0.1)$ difference between low and high PVS were tested for collinearity and were included in the regression model if the variance inflation factor was $<5$. Parameters with univariable significance $(\mathrm{P}<0.1)$ entered the multivariable regression analysis. Survival curves were constructed using Kaplan-Meier estimates and were compared by the log-rank test. To analyze the prediction of mortality, all baseline and procedural parameters that revealed significant $(\mathrm{P}<0.1)$ differences between patients with low and high PVS were tested for collinearity and were included in the regression model if the variance inflation factor was $<5$. Parameters with univariable significance $(\mathrm{P}<0.1)$ entered the multivariable Cox regression analysis. NT-proBNP serum levels did not enter either of these analyses due to the small sample size. All statistical analyses were performed using the SPSS statistical package version 26 (IBM Corp., Armonk, NY, USA).

\section{Results}

In the exploratory analysis, 1,445 patients with PVS $\leq-4$ \% (low PV, no volume overload) and 1,013 patients with PVS >-4 \% (high PV, volume overload) were identified (Figure 1 and Table 1). Patients with a high PVS were older, had a lower body mass index (BMI), worse renal function, and were more symptomatic. They also had a higher prevalence of coronary artery disease, higher NTproBNP serum levels, and a higher number of PCD. These characteristics resulted in higher EuroScores II calculated for patients with high PVS than for patients with low PVS.
$\mathrm{EF}$ and mean transvalvular gradients were not different between groups. Hemoglobin and hematocrit levels were lower in patients with high PVS. Accordingly, the percentage of patients diagnosed with anemia [according to the WHO (World Health Organization) definition as hemoglobin $(\mathrm{Hb})$ levels $<12.0 \mathrm{~g} / \mathrm{dL}$ in women and $<13.0 \mathrm{~g} / \mathrm{dL}$ in men (16)] was more than four times higher in patients with high PVS. Multivariable regression analysis revealed female sex, low BMI, a PCD and anemia as independent predictors of high PVS, with anemia showing the strongest impact (Table 2).

The use of balloon-expandable valves and the device success rates were not different between groups (Table 1). Periprocedural events according to VARC2 criteria (12) were more common among patients with high PVS without any predominance of any of the specific events (Table 1). This resulted in a slightly but significantly higher sum of VARC2 events in patients with a high PVS.

All-cause 30-day mortality in patients with high $v$ s. low PVS was $3.5 \%$ vs. $2.1 \%(\mathrm{P}=0.048)$, and cardiovascular 30 -day mortality was $3.1 \%$ vs. $1.9 \%(\mathrm{P}=0.074)$, respectively. Kaplan-Maier analysis (Figure $2 A$ ) revealed a twofold higher all-cause 1-year mortality rate for patients with high PVS than for those with low PVS (16.7 \% vs. $8.0 \%, \mathrm{P}<0.001)$; cardiovascular mortality was $11.4 \%$ vs. $5.6 \%(\mathrm{P}<0.001)$. When patients were stratified according to the presence of PCD (Figure 2B), Kaplan-Maier survival curves were not different than those depicting patients with high or low PVS, and 1-year mortality rates were $18.4 \%$ vs. 8.6\% $(\mathrm{P}<0.001)$ for $\mathrm{PCD} v s$. no $\mathrm{PCD}$, respectively.

The impact of significantly different baseline or procedural parameters on 1-year mortality rates in patients with high vs. low PVS was assessed by Cox regression analysis. One-year all-cause mortality was predicted by a low BMI, a high EuroSCORE II, PCD, and any VARC2 event, whereas high PVS was not an independent predictor (Table 3). When anemia was replaced by linear baseline hemoglobin levels, the latter parameter was found to be an additional independent predictor of mortality (Table 3).

High PVS was not correlated with the prevalence of PCD, as reflected by a $\varphi$ coefficient of 0.149 . Thus, PVS and PCD represent two different parameters of volume overload. Analysis of the ability of high PVS (>-4\%) to predict cardiac events (Table 4) showed that the sensitivity (35-59\%) and specificity (59-66\%) of PVS for diagnosing severely impaired functional NYHA status, a future event of cardiac decompensation, or (cardiovascular) death were low. The PPV of high PVS for the diagnosis of NYHA class 
Table 1 Patient characteristics and procedural data of the exploratory study population

\begin{tabular}{|c|c|c|c|}
\hline Characteristics & \multicolumn{2}{|c|}{ Entire study population } & $\mathrm{P}$ \\
\hline \multicolumn{4}{|l|}{ Demographic data } \\
\hline Female & $747(51.7)$ & $558(55.1)$ & 0.098 \\
\hline Age, y & 82.0. (780-85.0) & $83.0(79.0-86.0)$ & $<0.001$ \\
\hline GFR, $\mathrm{mL} / \mathrm{min} / 1.73 \mathrm{~m}^{2}$ & $70.0(50.0-88.0)$ & $60.0(43.0-82.0)$ & $<0.001$ \\
\hline Diabetes mellitus & $476(32.9)$ & $319(31.5)$ & 0.449 \\
\hline COPD & $283(19.6)$ & $183(18.1)$ & 0.344 \\
\hline NYHA class III/IV & $1,090(75.4)$ & $827(81.6)$ & $<0.001$ \\
\hline CABG & $153(10.6)$ & $116(11.5)$ & 0.500 \\
\hline Prior myocardial infarction & $166(11.5)$ & $130(12.8)$ & 0.313 \\
\hline Prior cardiac decompensation & $352(24.4)$ & $387(38.2)$ & $<0.001$ \\
\hline Atrial fibrillation & $597(41.3)$ & $419(41.4)$ & 0.993 \\
\hline Prior stroke & $176(12.2)$ & $134(13.2)$ & 0.441 \\
\hline Peripheral artery disease & $156(10.8)$ & $129(12.7)$ & 0.140 \\
\hline EuroScore II & $3.9(2.4-6.7)$ & $4.9(2.9-8.2)$ & $<0.001$ \\
\hline \multicolumn{4}{|l|}{ Laboratory Data } \\
\hline Actual PV, mL & $2,823.0(2,544.0-3,148.0)$ & $2,803.0(2,511.0-3,194.0)$ & 0.075 \\
\hline Ideal PV, mL & $3,200.0(2,840.0-3,600.0)$ & $2,730.0(2,418.0-3,042.0)$ & $<0.001$ \\
\hline Actual PVS, \% & $-10.6(-15.0--7.3)$ & $1.6(-1.3-6.0)$ & $<0.001$ \\
\hline $\mathrm{PV}_{\text {Duarte }}, \mathrm{mL} / \mathrm{g}$ & $4.40(4.00-4.84)$ & $5.79(5.25-6.55)$ & $<0.001$ \\
\hline \multicolumn{4}{|l|}{ Echocardiographic data } \\
\hline Ejection fraction, \% & $65.0(55.0-65.0)$ & $65.0(55.0-65.0)$ & 0.108 \\
\hline MPG, mmHg & $43.0(33.0-52.0)$ & $42.0(33.0-52.0)$ & 0.350 \\
\hline $\mathrm{MR} \geq 2+$ or $\mathrm{TR} \geq 2+$ & $213(14.7)$ & $220(21.7)$ & $<0.001$ \\
\hline \multicolumn{4}{|l|}{ Procedural data } \\
\hline Balloon-expandable valve & $555(38.4)$ & $360(35.6)$ & 0.153 \\
\hline Device success & $1309(90.6)$ & $902(89.0)$ & 0.210 \\
\hline
\end{tabular}

Table 1 (continued) 
Table 1 (continued)

\begin{tabular}{|c|c|c|c|}
\hline Characteristics & \multicolumn{2}{|c|}{ Entire study population } & $\mathrm{P}$ \\
\hline Any VARC2-defined event & $433(30.0)$ & $379(37.4)$ & $<0.001$ \\
\hline Major vascular complications & $95(6.6)$ & $92(9.1)$ & 0.021 \\
\hline Major bleeding events & $45(3.1)$ & $50(4.9)$ & 0.021 \\
\hline In-hospital stroke & $28(1.9)$ & $34(3.4)$ & 0.027 \\
\hline Residual aortic regurgitation $\geq \|^{\circ}$ & $24(1.8)$ & $45(4.8)$ & $<0.001$ \\
\hline
\end{tabular}

Data shown as number (\%) or median value (interquartile range). PVS, plasma volume status; PV, plasma volumes; BMI, body mass index; GFR, glomerular filtration rate (estimated); COPD, chronic obstructive pulmonary disease; NYHA, New York Heart Association; CAD, coronary artery disease; CABG, coronary artery bypass grafting; $\mathrm{VV}_{\text {Duarte }}$, plasma volume according to the formula of Duarte; MPG, mean pressure gradient; MR, mitral regurgitation; TR, tricuspid regurgitation; VARC2, Valve Academic Research Consortium-2.

Table 2 Binary logistic regression analysis with PVS >-4\% as dependent variable

\begin{tabular}{|c|c|c|c|c|}
\hline Variable & OR & \multicolumn{2}{|c|}{$95 \% \mathrm{Cl}$} & $\mathrm{P}$ \\
\hline Female sex & 2.546 & 1.951 & 3.323 & $<0.001$ \\
\hline BMI, $\mathrm{kg} / \mathrm{m}^{2}$ & 0.719 & 0.694 & 0.745 & $<0.001$ \\
\hline Prior cardiac decompensation & 1.496 & 1.131 & 1.978 & 0.005 \\
\hline
\end{tabular}

PVS, plasma volume state; OR, odds ratio; $\mathrm{Cl}$, confidence interval; BMI, body mass index.

A

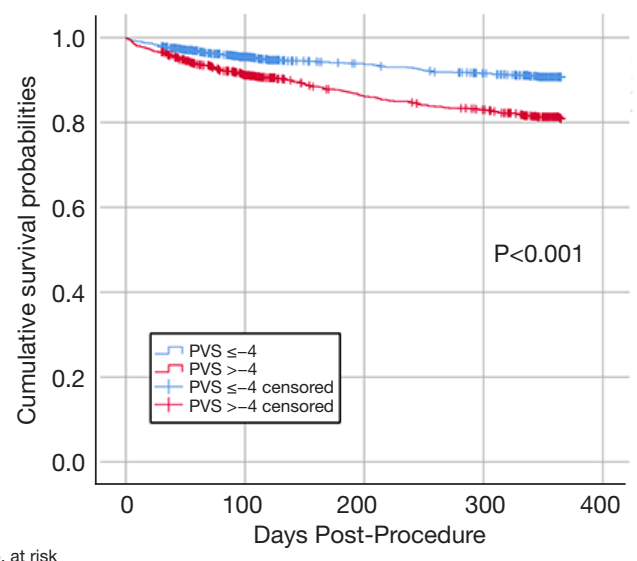

No. at risk

$\begin{array}{llllll}\text { PVS } \leq-4 & 1445 & 1184 & 1020 & 983 & 843\end{array}$

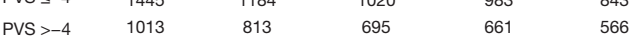

B

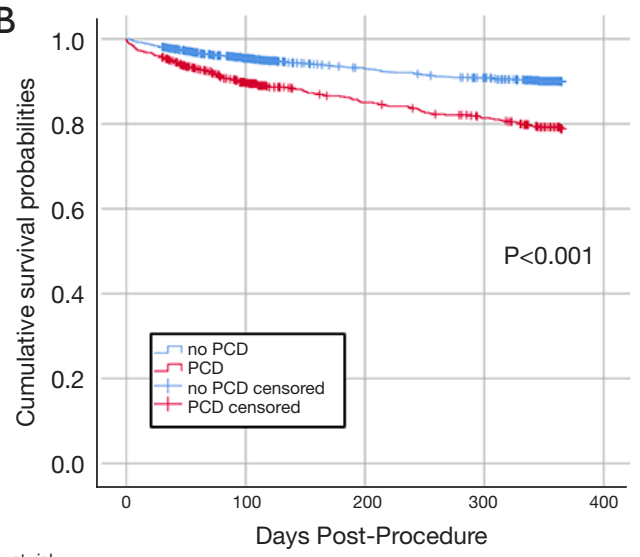

No. at risk

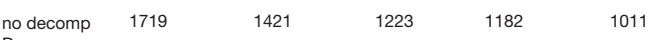

Figure 2 Survival curves based on pre-interventional PVS or PCD in the entire study population. Kaplan-Meier analysis of all-cause mortality of patients with (A) PVS (plasma volume status) $\leq-4 \%$ (low plasma volumes) vs. PVS >-4\% (high plasma volumes); (B) PCD (prior cardiac decompensation) before TAVI vs. no PCD. PVS, plasma volume status; PCD, prior cardiac decompensation; PVS, plasma volume status; TAVI, transcatheter aortic valve implantation. 
Table 3 Multivariable cox regression analysis with 1-year all-cause mortality as the outcome variable

\begin{tabular}{|c|c|c|c|c|}
\hline Variable & HR & \multicolumn{2}{|c|}{$95 \% \mathrm{Cl}$} & $\mathrm{P}$ \\
\hline \multicolumn{5}{|c|}{ Anemia included as a categorical variable } \\
\hline $\mathrm{BMI}, \mathrm{kg} / \mathrm{m}^{2}$ & 0.951 & 0.923 & 0.979 & 0.001 \\
\hline EuroSCORE II, \% & 1.042 & 1.027 & 1.058 & $<0.001$ \\
\hline VARC2 event & 2.326 & 1.839 & 2.942 & $<0.001$ \\
\hline \multicolumn{5}{|c|}{ Baseline hemoglobin included as a linear variable } \\
\hline BMI, kg/m² & 0.942 & 0.141 & 0.970 & $<0.001$ \\
\hline EuroSCORE II, \% & 1.044 & 1.028 & 1.060 & $<0.001$ \\
\hline VARC2 event & 2.294 & 1.813 & 2.903 & $<0.001$ \\
\hline
\end{tabular}

BMI, body mass index; HR, hazard ratio; Cl, confidence interval; VARC2, Valve Academic Research Consortium-2.

Table 4 Statistic performance characteristics of a PVS >-4\% or a PCD in predicting clinical events in the overall study population (n=2,458)

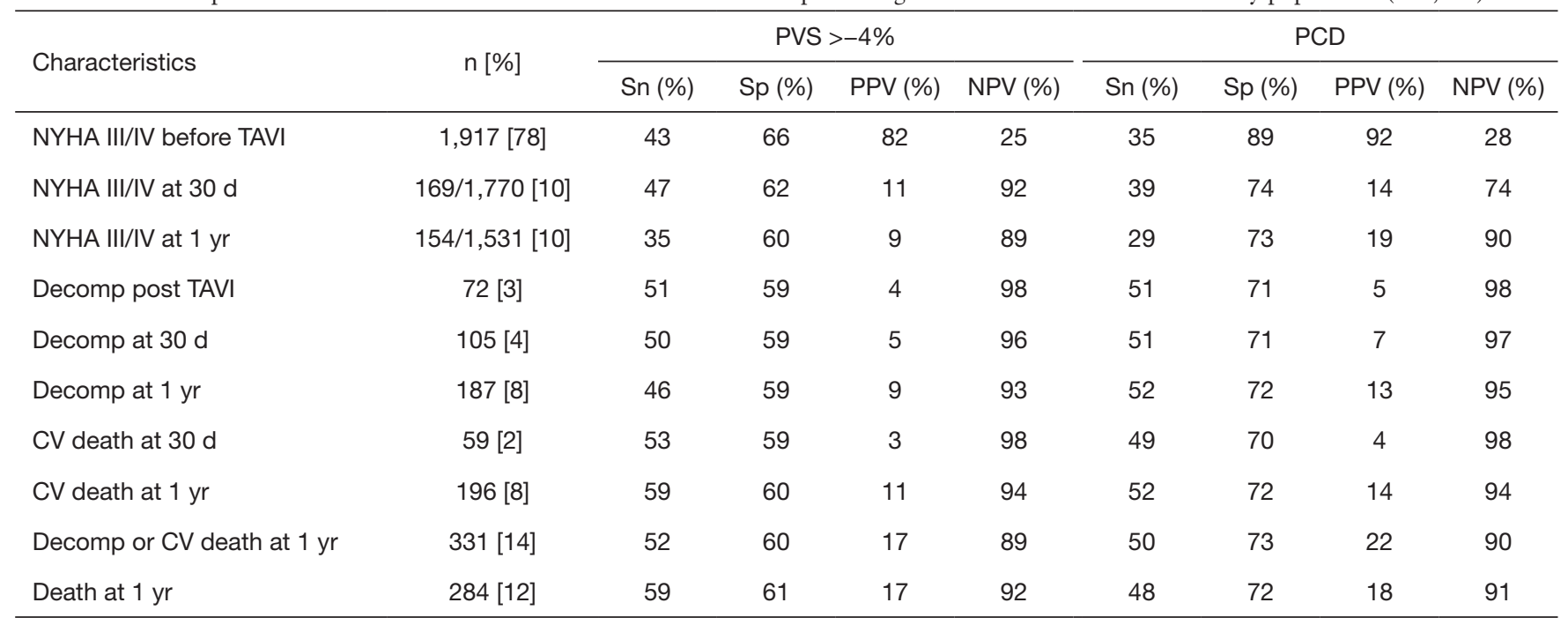

PVS, plasma volume state; PCD, prior cardiac decompensation; Sn, sensitivity; Sp, specificity; PPV, positive predictive value; NPV, negative predictive value; Decomp, cardiac decompensation; CV, cardiovascular.

III/IV before TAVI was high (82\%) but very low (3-17\%) for all future events. The NPV (89-98\%) of high PVS was revealed to be a useful parameter for excluding any events post-TAVI. Analysis of PCD showed that this parameter had a prognostic ability that was similar to that of high PVS; however, the specificity (70-89\%) of PCD was higher (Table 4).

We identified 1512 patients with HG-AS (controls), 239 patients with LFLG-AS, and 327 patients with pLFLGAS (Figure 1 and Table 5). Compared with patients with HG-AS, patients with LFLG-AS were characterized by a significantly higher number of manifest cardiovascular diseases and were at significantly higher calculated risk as reflected by a median EuroScore II of 9.3\% (5.6-16.0\%), clearly signifying that these patients belonged to a highrisk population. EF and MPG, per definition, were 
Table 5 Patient characteristics of the LFLG-AS study populations

\begin{tabular}{|c|c|c|c|c|}
\hline Characteristics & Control, $n=1,512$ & LFLG-AS, n=239 & pLFLG-AS, n=327 & $\mathrm{P}$ \\
\hline Female & $845(55.9)$ & $70(23.9)$ & $186(56.9)$ & $<0.001$ \\
\hline Age, y & $82.0(79.0-85.0)$ & $80.0(76.0-84.0)$ & $82.0(79.0-86.0)$ & $<0.001$ \\
\hline $\mathrm{BMI}, \mathrm{kg} / \mathrm{m}^{2}$ & $26.8(24.2-30.8)$ & $26.8(24.2-29.7)$ & $27.5(24.4-31.2)$ & 0.153 \\
\hline COPD & $293(19.4)$ & $34(14.2)$ & $67(20.5)$ & 0.125 \\
\hline NYHA class III/IV & $1,144(75.7)$ & $205(85.8)$ & $265(81.0)$ & 0.001 \\
\hline \multicolumn{5}{|l|}{ Cardiovascular disease } \\
\hline Atrial fibrillation & $286(20.9)$ & $105(48.8)$ & $157(53.6)$ & $<0.001$ \\
\hline Prior stroke & $166(11.0)$ & $30(12.6)$ & $60(18.3)$ & 0.001 \\
\hline Peripheral artery disease & $146(9.7)$ & 45 (18.8) & $49(15.0)$ & $<0.001$ \\
\hline Euro Score II & $3.5(2.3-5.9)$ & $9.3(5.6-16.0)$ & $5.5(3.4-8.7)$ & $<0.001$ \\
\hline \multicolumn{5}{|l|}{ Laboratory data } \\
\hline NT-proBNP, ng/L & $1,658.0(673.0-4,774.0)$ & $6,794.0(3,089.0-14,634.0)$ & $2,132.0(874.0-3,937.0)$ & $<0.001$ \\
\hline $\mathrm{n}$ & 335 & 62 & 85 & \\
\hline Hemoglobin, g/dL & $12.7(11.6-13.7)$ & $12.8(11.4-14.4)$ & $12.7(11.6-13.8)$ & 0.091 \\
\hline \multicolumn{5}{|l|}{ Echocardiographic data } \\
\hline Ejection fraction, \% & $65.0(60.0-65.0)$ & $35.0(25.0-40.0)$ & $65.0(55.0-65.0)$ & $<0.001$ \\
\hline MPG, mmHg & $49.0(43.0-59.0)$ & $24.0(17.0-32.0)$ & $28.0(22.0-34.0)$ & $<0.001$ \\
\hline $\mathrm{MR} \geq 2+$ or $\mathrm{TR} \geq 2+$ & $231(15.3)$ & $89(37.2)$ & $67(20.5)$ & $<0.001$ \\
\hline \multicolumn{5}{|l|}{ Procedural data } \\
\hline Balloon-expandable valve & $572(37.9)$ & $112(46.9)$ & $114(34.9)$ & 0.010 \\
\hline Device success & $1,328(87.8)$ & $220(92.1)$ & $306(93.6)$ & 0.003 \\
\hline Any VARC2-defined event & $539(35.6)$ & $82(34.3)$ & $85(26.0)$ & 0.004 \\
\hline Residual aortic regurgitation $\geq \mathrm{II}^{\circ}$ & $45(3.2)$ & $6(2.6)$ & $10(3.3)$ & 0.901 \\
\hline
\end{tabular}

Data shown as number (\%) or median value (interquartile range). LFLG-AS, low-flow low-gradient aortic stenosis; pLFLG-AS, paradoxical LFLG-AS; BMI, body mass index; GFR, glomerular filtration rate (estimated); COPD, chronic obstructive pulmonary disease; NYHA, New York Heart Association; CAD, coronary artery disease; CABG, coronary artery bypass grafting; PVS, plasma volume status; $P V_{\text {Duarte }}$, plasma volume according to the formula of Duarte; MPG, mean pressure gradient; MR, mitral regurgitation; TR, tricuspid regurgitation; VARC2, Valve Academic Research Consortium-2. 
A

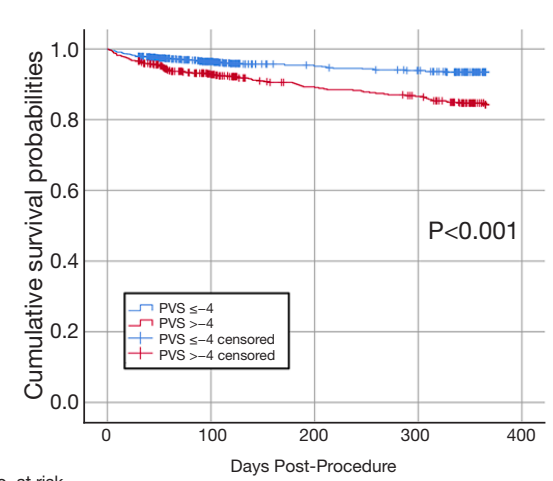

$\begin{array}{llllll}\text { No. at risk } & & & & & \\ \text { PVS } \leq-4 & 899 & 730 & 629 & 614 & 529 \\ \text { PVS }>-4 & 613 & 491 & 430 & 413 & 356\end{array}$

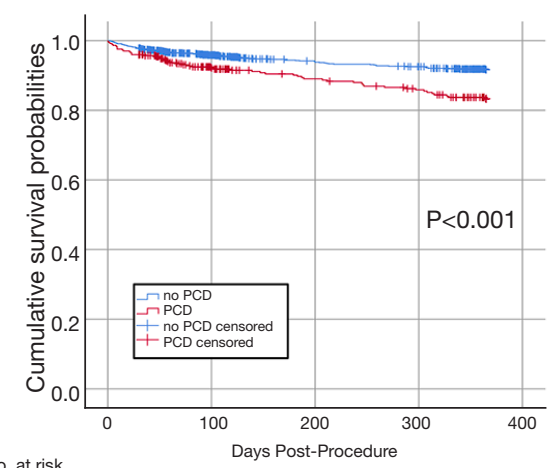

No. at risk

$\begin{array}{llllll}\text { no decomp } & 1140 & 934 & 805 & 787 & 679\end{array}$
B

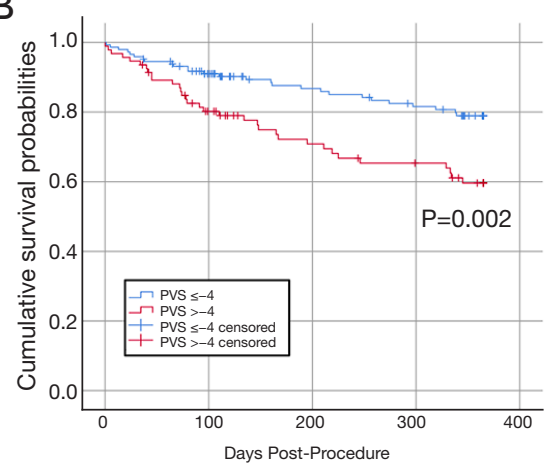

146
93

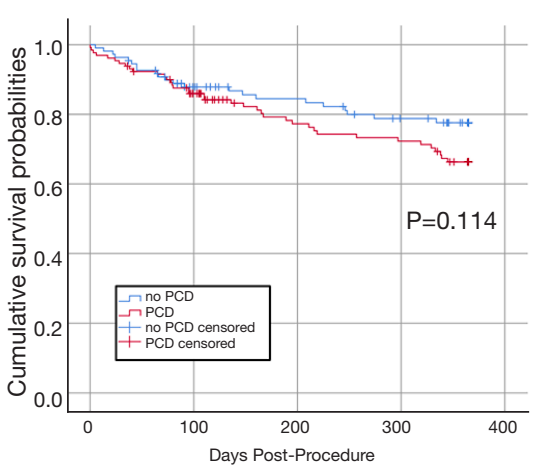

$\begin{array}{lcccc}109 & 86 & 75 & 66 & 57 \\ 130 & 103 & 78 & 73 & 63\end{array}$

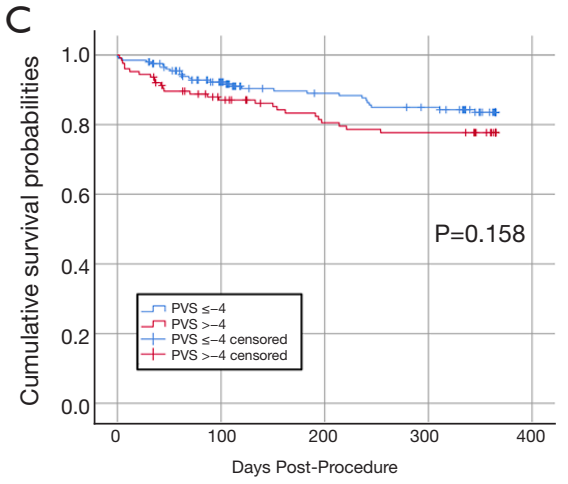

$\begin{array}{llccc}201 & 161 & 132 & 124 & 102 \\ 126 & 100 & 86 & 83 & 74\end{array}$

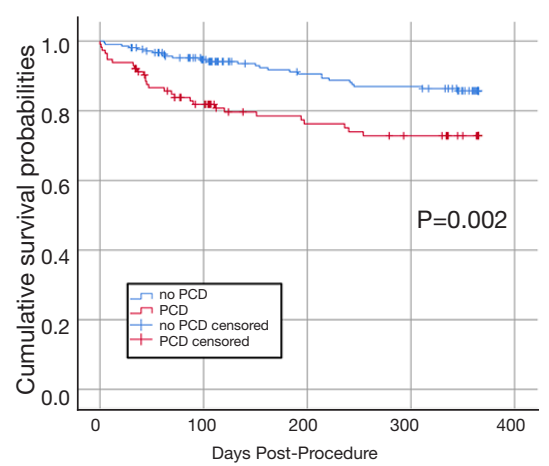

$\begin{array}{lllll}213 & 179 & 151 & 145 & 122\end{array}$

Figure 3 Survival curves based on the pre-interventional PVS or PCD in patients with LFLG-AS. Kaplan-Meier analysis of all-cause mortality of patients with (A) HG-AS, (B) LFLG-AS and (C) pLFLG-AS. Upper panels: Survival curves based on PVS $\leq-4 \%$ (low plasma volumes) vs. PVS >-4\% (high plasma volumes). Lower panels: survival curves based on PCD (prior cardiac decompensation) before TAVI $v s$. no PCD. PVS, plasma volume status; PCD, prior cardiac decompensation; LFLG-AS, low-flow low-gradient aortic stenosis; HG-AS, highgradient aortic stenosis; pLFLG-AS, paradoxical low-flow, low-gradient aortic stenosis; TAVI, transcatheter aortic valve implantation.

substantially lower in LFLG-AS patients. These differences were associated with a higher percentage of these patients being in NYHA class III or IV and higher NT-proBNP serum levels. Values for baseline characteristics of patients with pLFLG-AS were generally between those of patients with HG-AS and patients with LFLG-AS, and pLFLGAS patients had an intermediate EuroScore II of 5.5\% (3.4-8.7\%). Hemoglobin levels and the prevalence of anemia were not different between groups. Hematocrits were slightly higher in patients with low-gradient AS compared with controls $(\mathrm{P}=0.010)$. The calculated absolute plasma volumes and the percentage of patients with a relative plasma volume overload indicated by PVS $>-4 \%$ showed no differences between the three groups. The $\mathrm{PV}_{\text {Durte }}$ levels estimated by an alternative formula tended to be even lower in patients with LFLG-AS compared to the two other groups $(\mathrm{P}=0.039)$. The device success rates were highest and the VARC2-defined periprocedural events were lowest in patients with pLFLG-AS.

The prognostic impact of two pre-interventional parameters of cardiac congestion (PVS vs. PCD) on survival was investigated in the three subgroups. In control patients (HG-AS), high PVS (Figure $3 A$ ) and a history of PCD (Figure 3B) had a similar impact on all-cause 1-year mortality, with mortality rates of $13.7 \%$ vs. $5.7 \%$ (high vs. low PVS, $\mathrm{P}<0.001$ ) and $14.2 \%$ vs. $7.2 \%$ (PCD vs. no PCD, $\mathrm{P}<0.001$ ). In LFLG-AS patients (Figure $3 B$ ) the respective mortality rates were $35.5 \%$ vs. $18.5 \%(\mathrm{P}=0.002)$ and 29.2 vs. $20.2(\mathrm{P}<0.114)$. In pLFLG-AS patients (Figure $3 C)$, mortality rates were $20.6 \%$ vs. $13.9 \%(\mathrm{P}=0.158)$ and 24.6 vs. $12.2 \%(\mathrm{P}=0.002)$, respectively.

The performance of high PVS in predicting outcomes 
was analyzed in all three subpopulations. Although patients in the LFLG-AS and pLFLG-AS groups remained more symptomatic after TAVI, had more episodes of cardiac decompensation, and had a higher mortality rate, the predictive value of PVS was similar for the three groups and was comparable to the results for the study population overall: PVS $>-4 \%$ had a low sensitivity, a slightly higher specificity, and very low PPV but high NPV.

\section{Discussion}

We found that PVS >-4\%-as marker of latent volume overload-and at least one episode of pre-interventional PCD_indicating an event of overt volume overloademerged as two independent parameters with an almost identical negative impact on 1-year prognosis in patients undergoing transfemoral TAVI for severe AS. However, in contrast to PCD, high PVS was related to low hemoglobin levels and lost its prognostic significance when corrected for anemia. Both parameters had a low sensitivity and specificity for predicting adverse events post-TAVI, but a negative test result before TAVI had a high NPV to rule out further events. Interestingly, PVS was not different between patients with high-gradient AS and those with LFLG-AS or pLFLG-AS, which excludes the hypothesis that latent volume overload plays a role in the latter highrisk populations. Predictive values of high PVS were similar in all three subgroups compared with that of the overall population. Finally, Kaplan-Maier analyses revealed similar 1-year mortality rates for high PVS and the presence of PCD within all three subgroups. Taken together, PVS in our patient population is associated with hemoglobin levels, does not unmask latent volume overload in patients with LFLG-AS, and has no statistical superiority in predicting future cardiovascular events compared with PCD.

Three recently published studies addressed the role of PVS in patients undergoing TAVI using the same formula as in our study (8-10). Although patients of these studies differed from ours with respect to sample size and the fact that $16-42 \%$ of the patients with non-transfemoral (mostly apical) access routes were included, they all reported similar results: patients with a high PVS were characterized by a higher cardiovascular baseline risk, a higher rate of inhospital adverse events and a higher long-term mortality rate. Only two of these studies reported hemoglobin values and these data clearly demonstrate an inverse relationship between high PVS values and low hemoglobin levels. However, statistical maneuvers to correct for these crucial differences between baseline parameters vary largely among studies and while the authors discussed a potential role of anemia in patients with a high PVS, they did not correct for this parameter in further analyses. Therefore, the role of anemia remains largely unclear in these studies. Our analyses unmasked a clear association between low hemoglobin (and hematocrit) levels and PVS, an impact of anemia that clearly obscured any prognostic value of PVS. Indeed, the prevalence of anemia in patients with stable HF (with reduced or preserved EF) is as high as $30 \%$ and that of hospitalized patients $50 \%$ (17), and patients with $\mathrm{HF}$ and anemia have markedly increased rates of hospitalization and death $(17,18)$. Therefore, one could speculate that high PVS in our population is rather a surrogate marker of anemia than of volume overload. In addition to iron deficiency, renal disease, inflammation, or myeloproliferative status, hemodilution might cause anemia in patients with HF, which brings us back to the starting point of PVS as a marker of subclinical volume overload. More detailed blood cell analyses are needed in order to distinguish between anemia caused mainly by hemodilution and that due to other, genuine pathologies.

Our analyses of the role of PVS as an indicator of shortterm mortality and for the occurrence of VARC2-defined periprocedural adverse events are in line with previous results in patients undergoing coronary bypass surgery (7) or TAVI (8-10) that demonstrated a negative association between high PVS and in-hospital adverse events. However, an interesting finding of our study is the lack of an association of PVS before TAVI with future HF events, as reflected by low sensitivities and specificities. Only the NPV emerged as a useful parameter to exclude such events; however, NPV and PPV hinge on the prevalence of disease, and the high NPV in our study might be dependent on the rather low event rates, hampering the generalization of these results.

On the other hand, it is notable that PCD is similarly prognostic of outcomes. The impact of an event of worsening HF prior to TAVI on short- and mid-term outcomes after TAVI, including future hospitalizations for HF, was demonstrated in a recent analysis of the Placement of Aortic Transcatheter Valves (PARTNER) 2 trial and registries (19). These results, together with abundant previous data $(4,20-22)$, confirm the hypothesis that concomitant, established HF persists in certain patients with AS, even after restoration of aortic valve function by TAVI. In our study, it was even more surprising to see the parallel prognostic impacts of high PVS (immediately before TAVI) and the existence of PCD, since the definition 
of PCD in our study was not restricted to a particular time period before TAVI. This finding underlines once more the critical "red flag" character of any cardiac decompensation in the course of HF and may have impact on the urgency of correction of high afterload by TAVI in these patients.

Patients with LFLG-AS present not only with clinical symptoms and signs of HF but also display compromised hemodynamic performance (low stroke volume, impaired LV function, high natriuretic peptide levels). These patients have an increased calculated perioperative risk, and, indeed, experience significantly higher event rates post-TAVI as well. Therefore, the lack of differences in calculated PV among patient populations with or without LFLG-AS is an unexpected finding. Patients' actual PV and the percentage of those with PVS >-4\% were not different between groups. In addition, $\mathrm{PV}_{\text {Duarte, }}$, calculated by a different method that thereby excludes potential interferences between body weight, congestion, and HF-related cardiac cachexia (23), revealed no meaningful differences between groups. Accordingly, the ability of PVS to predict outcomes in these subgroups was likewise limited. One can only speculate whether medical effects in patients with LFLG-AS might obscure the significance of PVS in these apparently compromised patient populations. It is conceivable that patients who exhibit objective signs of HF, but in whom the diagnosis of severe AS may be delayed due to the low-flow status, are given a stricter ambulatory diuretic treatment before TAVI. This might explain the slightly higher hematocrit levels in patients with LG-AS compared with controls and may have led to lower values for calculated PV. Following this line of argumentation, it is not surprising that such a treatment that is purely symptom-based-and, accordingly, those parameters influenced by this treatment like PVS-does not translate into any prognostic benefit. Clearly, the patients' medical treatment should be analyzed in detail to shed more light on these interesting interactions.

One limitation of this study is that the information concerning cardiac decompensation (prior to or after TAVI) was derived from medical reports, patients' anamnesis, or follow-up visit examinations; thus, we are not able to provide more detailed information on clinical parameters (pulmonary and/or peripheral edema, blood pressures, diuresis, etc.) that would allow a glimpse into hemodynamic mechanisms. The potential interference of concomitant medical therapy may have influenced our results.

In conclusion, although high PVS, indicative of volume overload, identified patients with AS facing a worse 1-year survival even after least-invasive aortic valve replacement by transfemoral TAVI, PVS did not appear to be a useful parameter to predict future cardiac decompensations, worsening functional status, or short-term cardiovascular mortality post-TAVI. Moreover, the similar PVS levels among control patients with high-gradient AS and those with LFLG-AS suggest there was a similar calculated volume load in all patients despite higher calculated risks, higher rates of manifest cardiovascular diseases, and higher rates of follow-up events in the latter population. It remains unclear whether the strikingly high prevalence of anemia in patients with high PVS is caused by hemodilution or if anemia of other etiologies leads to high calculated PVS levels. A report of a PCD occurring before TAVI, taken simply from a patient's history, had a prognostic value in predicting future events that was similar to that of preinterventional PVS.

\section{Acknowledgments}

We thank Elizabeth Martinson, PhD, from the KHFI Editorial Office for excellent editorial work.

Funding: None.

\section{Footnote}

Reporting Checklist: The authors have completed the STROBE reporting checklist. Available at https://dx.doi. org/10.21037/cdt-21-286

Data Sharing Statement: Available at https://dx.doi. org/10.21037/cdt-21-286

Conflicts of Interest: All authors have completed the ICMJE uniform disclosure form (available at https://dx.doi. org/10.21037/cdt-21-286). MR receives speaker fees from Abbott. CL receives lecture, consulting fees and/ or support for attending meetings and/or travel from Abbott, Astra Zeneca, Bayer, Berlin Chemie, Boehringer lngelheim, Daiichi-Sankyo, Pfizer-Bristol-Myers Squibb, Thermo Fisher, Xenios AG. Yeong-Hoon Choi receives proctor/speaker/scientific advisor fees from Getinge, Jotec/ CryoLife, Cytosorbents. CWH is on advisory board of Medtronic. WKK receives proctor/advisory board/speaker fees from Abbott, Boston Scientific, Edwards Lifesciences, Medtronic, Meril Life Sciences, Shockwave Medical. The other authors have no conflicts of interest to declare.

Ethical Statement: The authors are accountable for all 
aspects of the work in ensuring that questions related to the accuracy or integrity of any part of the work are appropriately investigated and resolved. The study was conducted in accordance with the Declaration of Helsinki (as revised in 2013). The study was approved by institutional ethics committee of the University of Giessen (No. AZ 01/21) and individual consent for this retrospective analysis was waived.

Open Access Statement: This is an Open Access article distributed in accordance with the Creative Commons Attribution-NonCommercial-NoDerivs 4.0 International License (CC BY-NC-ND 4.0), which permits the noncommercial replication and distribution of the article with the strict proviso that no changes or edits are made and the original work is properly cited (including links to both the formal publication through the relevant DOI and the license). See: https://creativecommons.org/licenses/by-nc-nd/4.0/.

\section{References}

1. Yerasi C, Tripathi B, Wang Y, et al. National trends and 30-day readmission rates for next-day-discharge transcatheter aortic valve replacement: An analysis from the Nationwide Readmissions Database, 2012-2016. Am Heart J 2021;231:25-31.

2. Durand E, Doutriaux M, Bettinger N, et al. Incidence, Prognostic Impact, and Predictive Factors of Readmission for Heart Failure After Transcatheter Aortic Valve Replacement. JACC Cardiovasc Interv 2017;10:2426-36.

3. Arora S, Hendrickson MJ, Strassle PD, et al. Trends in Costs and Risk Factors of 30-Day Readmissions for Transcatheter Aortic Valve Implantation. Am J Cardiol 2020;137:89-96.

4. Fischer-Rasokat U, Renker M, Liebetrau C, et al. Outcome of patients with heart failure after transcatheter aortic valve implantation. PLoS One 2019;14:e225473.

5. Abrahamsson P, Swedberg K, Borer JS, et al. Risk following hospitalization in stable chronic systolic heart failure. Eur J Heart Fail 2013;15:885-91.

6. Ling HZ, Flint J, Damgaard M, et al. Calculated plasma volume status and prognosis in chronic heart failure. Eur J Heart Fail 2015;17:35-43.

7. Maznyczka AM, Barakat MF, Ussen B, et al. Calculated plasma volume status and outcomes in patients undergoing coronary bypass graft surgery. Heart 2019;105:1020-6.

8. Adlbrecht C, Piringer F, Resar J, et al. The impact of subclinical congestion on the outcome of patients undergoing transcatheter aortic valve implantation. Eur J Clin Invest 2020. [Epub ahead of print]. doi: 10.1111/ eci.13251.

9. Maznyczka AM, Barakat M, Aldalati O, et al. Calculated plasma volume status predicts outcomes after transcatheter aortic valve implantation. Open Heart 2020;7:e001477.

10. Shimura T, Yamamoto M, Yamaguchi R, et al. Calculated plasma volume status and outcomes in patients undergoing transcatheter aortic valve replacement. ESC Heart Fail 2021;8:1990-2001.

11. Fischer-Rasokat $\mathrm{U}$, Renker M, Liebetrau C, et al. 1-Year Survival After TAVR of Patients With Low-Flow, LowGradient and High-Gradient Aortic Valve Stenosis in Matched Study Populations. JACC Cardiovasc Interv 2019;12:752-63.

12. Kappetein AP, Head SJ, Généreux P, et al. Updated standardized endpoint definitions for transcatheter aortic valve implantation: the Valve Academic Research Consortium-2 consensus document (VARC-2). Eur J Cardiothorac Surg 2012;42:S45-60.

13. Longo D, Fauci A, Kasper D, et al. Harrison's Principles of Internal Medicine, Table 218. 18th edition ed. New York: McGraw-Hill Education, 2011.

14. Kobayashi M, Girerd N, Duarte K, et al. Prognostic impact of plasma volume estimated from hemoglobin and hematocrit in heart failure with preserved ejection fraction. Clin Res Cardiol 2020;109:1392-401.

15. Duarte K, Monnez JM, Albuisson E, et al. Prognostic Value of Estimated Plasma Volume in Heart Failure. JACC Heart Fail 2015;3:886-93.

16. Nutritional anaemias. Report of a WHO scientific group. World Health Organ Tech Rep Ser 1968;405:5-37.

17. Anand IS, Gupta P. Anemia and Iron Deficiency in Heart Failure: Current Concepts and Emerging Therapies. Circulation 2018;138:80-98.

18. Paolillo S, Scardovi AB, Campodonico J. Role of comorbidities in heart failure prognosis Part I: Anaemia, iron deficiency, diabetes, atrial fibrillation. Eur J Prev Cardiol 2020;27:27-34.

19. Chen S, Redfors B, Crowley A, et al. Impact of recent heart failure hospitalization on clinical outcomes in patients with severe aortic stenosis undergoing transcatheter aortic valve replacement: an analysis from the PARTNER 2 trial and registries. Eur J Heart Fail 2020;22:1866-74.

20. Jalava MP, Laakso T, Virtanen M, et al. Transcatheter and Surgical Aortic Valve Replacement in Patients With Recent Acute Heart Failure. Ann Thorac Surg 2020;109:110-7. 
21. Vassileva CM, Telila T, Markwell S, et al. Magnitude of negative impact of preoperative heart failure on mortality during aortic valve replacement in the medicare population. Ann Thorac Surg 2015;99:1503-09; discussion 1509-10.

22. Thaden JJ, Balakrishnan M, Sanchez J, et al. Left ventricular filling pressure and survival following aortic

Cite this article as: Fischer-Rasokat $\mathrm{U}$, Renker M, Liebetrau C, Weferling M, Rieth A, Rolf A, Choi YH, Hamm CW, Kim WK. Predictive value of overt and non-overt volume overload in patients with high- or low-gradient aortic stenosis undergoing transcatheter aortic valve implantation. Cardiovasc Diagn Ther 2021;11(5):1080-1092. doi: 10.21037/cdt-21-286 valve replacement for severe aortic stenosis. Heart 2020;106:830-7.

23. Anker SD, Negassa A, Coats AJ, et al. Prognostic importance of weight loss in chronic heart failure and the effect of treatment with angiotensin-converting-enzyme inhibitors: an observational study. Lancet 2003;361:1077-83. 